\title{
Short communication: Comparative proteomic analysis of the lactating and nonlactating bovine mammary gland
}

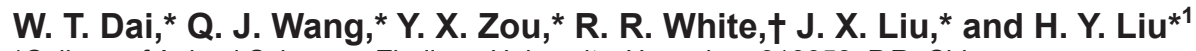 \\ ${ }^{*}$ College of Animal Sciences, Zhejiang University, Hangzhou 310058, P.R. China \\ †Department of Dairy Science, Virginia Tech, Blacksburg 24060
}

\begin{abstract}
The objective of this study was to determine how bovine mammary protein profiles vary during lactation and the dry period. Three lactating and 3 nonlactating cows were selected for mammary gland tissue sampling. Compared with the mammary proteins in nonlactating cows, a total of 60 differentially expressed proteins (DEP, including 57 upregulated and 3 downregulated) were identified in lactating cows using 2-dimensional difference gel electrophoresis combined with mass spectrometry. These DEP included enzymes and proteins associated with various macromolecular metabolic processes, and appeared to promote the increased metabolic activity associated with milk synthesis and secretion. The increased DEP were primarily related to initiation, maintenance, and involution of lactation, and included proteins involved in glycolysis/gluconeogenesis, the tricarboxylic acid cycle, the pentose phosphate pathway, oxidative phosphorylation, aminoacyltransfer RNA biosynthesis, and fatty acid biosynthesis. Identified DEP were further validated by real-time, reverse-transcription PCR and Western blot. Five new DEP associated with lactation were uniquely identified. This work provided some protein-associated insights to facilitate further investigation of the mechanisms underlying lactation in dairy cows.
\end{abstract}

Key words: lactation, bovine mammary gland, 2-dimensional proteomics

\section{Short Communication}

The bovine mammary gland (MG) is a specialized organ designed to synthesize and secrete large quantities of milk (Jena et al., 2015). The bovine MG is also characterized by its successive pregnancy-lactation involution cycle (Desrivieres et al., 2007), during which the

Received November 28, 2016.

Accepted March 6, 2017.

${ }^{1}$ Corresponding author: hyliu@zju.edu.cn functional development of MG is hormonally regulated (Bellmann, 1976) and involves complex shifts in proteins (Jena et al., 2015). The MG of dry cows provides a baseline for the expression of enzymes and proteins at the onset of lactation (Hurley, 1989). During late gestation, the MG prepares for lactation by responding to prolactin signaling (Bellmann, 1976), the pathway of which then induces milk protein gene and biosynthetic enzyme expression to promote lactogenesis and lactation maintenance (Yang et al., 2000). Numerous early studies focused on the molecular dynamics of the MG under differing genetic, physiologic, and morphologic conditions. These studies have focused on protein and lipid synthesis during the lactation cycle (Bionaz and Loor, 2008, 2011), transcriptional differences associated with development and lactation ( $\mathrm{Li}$ et al., 2012, 2016; Suarez-Vega et al., 2016), and immunological changes associated with mastitis (Huang et al., 2014; Zhao et al., 2015). Several groups have studied the proteomic profile of large ruminant MG during lactation, investigating shifts in carbohydrate and lipid metabolism during lactation in Bos taurus (Beddek et al., 2008), metabolic shifts in the MG associated with stage of lactation (Rawson et al., 2012), and MG profiles of buffaloes during lactation and the dry period (Jena et al., 2015). However, alterations in proteins related to lactation, as identified by comparing lactating and nonlactating bovine MG, have not been studied extensively with proteomic approaches. Therefore, the aim of this study was to screen for the differentially expressed proteins (DEP) associated with lactation in the bovine MG.

Experimental procedures were approved by the Institutional Animal Care and Use Committee at Zhejiang University, Zhejiang, China. Six multiparous and healthy, mastitis-free, Chinese Holstein cows housed at the Hangjiang Dairy Farm (Hangzhou, China) were selected for the study. Three cows in dry period (52 \pm 7 mo of age; mean \pm SD) and 3 cows in mid-lactation (58 \pm 7 mo of age; 92 to 118 DIM) were slaughtered as described in a previous study (Dufour and Roy, 1985). Samples of 6 MG tissues were collected immediately 
after slaughter following the methods described by Wang et al. (2016). The 50-mg aliquots of MG tissue were homogenized in $0.25 \mathrm{~mL}$ of lysis buffer for $1 \mathrm{~h}$ on ice as described in $\mathrm{Wu}$ et al. (2012). The homogenates were centrifuged at $13,000 \times g$ for $5 \mathrm{~min}$ at $4^{\circ} \mathrm{C}$, the supernatants were then removed and proteins were collected. Protein concentrations were determined using the Bradford method (Bradford, 1976). Each $200 \mu \mathrm{g}$ of MG proteins derived from individual samples were resolved in individual 2-dimensional $(\mathbf{2 - D})$ gels $(\mathrm{n}=3$, gels derived from 3 individual MG of 3 lactating and nonlactating groups, respectively). The procedure for $2-\mathrm{D}$ electrophoresis was performed as described by $\mathrm{Wu}$ et al. (2012). Image Master 2D Platinum (GE Healthcare, Pittsburgh, PA) software was used for matching and analysis of protein spots in 2-D gels. Prior to analysis, all gel images were cropped to identical sizes by removing areas extraneous to the proteins spots using PDQuest 2D analysis software (version 8.0, Bio-Rad, Hercules, CA). Only the spots that were present in all gels and those that were absent from a maximum of one analytical gel per group were considered for the statistical comparison. Differential intensity levels of corresponding protein spots in the 2 treatments were analyzed by unpaired Student's $t$-test. Only the significantly expressed protein spots $(P<0.05)$ with a 2.0 -fold change or more in intensity were selected for MS identification as described by Wu et al. (2012).

The gene primers (Supplemental Table S1; https:// doi.org/10.3168/jds.2016-12366) were designed using DNAStar software (DNASTAR, Madison, WI). Total RNA was isolated from each MG sample using an RNeasy Mini Kit (Qiagen, Hilden, Germany) and then reverse transcribed to cDNA using a SYBR PrimeScript RT-PCR Kit (Takara, Tokyo, Japan). The mRNA abundance of these genes was analyzed by quantitative real-time PCR (qRT-PCR) using SYBR Green as described previously (Zhao et al., 2010). The relative mRNA abundance was calculated using the $2^{-\Delta \Delta \mathrm{Ct}}$ method (Schmittgen and Livak, 2008) and normalized to $\beta$-actin mRNA in the same sample. Three replicates were performed in each sample.

The Western analysis of MG proteins [eukaryotic translation initiation factor 2 subunit 1 (EIF2S1), kappa-casein (CSN3), and $\beta$-actin] were performed as previously described in Yang et al. (2015). All the primary antibodies were purchased from Abcam (Cambridge, MA), validated with bovine samples before use, and diluted at 1:1,000 in PBS. The relative quantity of protein bands was determined by ImagePro Plus software (Media Cybernetics, Rockville, MD) and normalized to $\beta$-actin protein in the same sample. The normalized data of qRT-PCR and Western blot were analyzed by Student's $t$-test. Differences were considered statistically significant at $P<0.05$.

The stage of lactation is one of the key drivers of milk production and milk component yields (McManaman and Neville, 2003), and a dry period of sufficient length (about $60 \mathrm{~d}$ ) is essential for mammary cell turnover to optimize milk production following lactation (Collier et al., 2012). The mammary physiology and metabolism undergo a wide range of dynamic changes as the animal transitions from the nonlactating to lactating states (Hurley, 1989). Therefore, it is important to identify key protein alterations and molecular mechanisms underlying this transition. Compared with nonlactating bovine MG, we detected 80 DEP spots corresponded to 60 unique proteins (Supplemental Table S2; https:// doi.org/10.3168/jds.2016-12366) that were differentially abundant in the lactating group. Of these proteins, 57 were upregulated (Supplemental Figure S1A; https://doi.org/10.3168/jds.2016-12366) and 3 were downregulated (Supplemental Figure S1B; https:// doi.org/10.3168/jds.2016-12366). More than half of these DEP were enzymes functioning in AA, protein, lipid, and nucleotide metabolism, and their differential expression was consistent with previous studies of the lactating bovine MG (Rudolph et al., 2007; Bionaz and Loor, 2011; Rawson et al., 2012). The milk proteins $\alpha_{\mathrm{S} 2}$-casein and CSN3 were significantly increased in the lactating MG; however, other casein proteins (such as $\alpha_{S 1}$-isoforms and $\beta$-isoforms) were not observed. Variation in 2-D proteomic analysis via gel exercising followed by MS identification may account for the absence of these casein isoforms.

Some DEP that have not been reported before in the MG during lactation were detected, including mitochondrial inner membrane protein (IMMT), 3-hydroxyisobutyrate dehydrogenase (HIBADH), dimethylarginine dimethylaminohydrolase 1 (DDAH1), $\mathrm{N}$-acetylneuraminic acid synthase (NANS), endoplasmic reticulum lectin 1 (ERLEC1), and sec13 protein homolog (SEC13). The protein DDAH1 is an enzyme that metabolizes methylated arginine to citrulline and methylamine, which regulates organ development (in brain, kidney, and gastrointestinal tissues) in adult rats and chickens (Mishima et al., 2004; Breckenridge et al., 2010). Although its definite function in mammary development has not been elucidated, the upregulation of DDAH1 $(P=0.0011)$ within the lactating MG might be related to mammary differentiation. Also, the increased protein SEC13 is mainly involved in the biogenesis of coat protein complex II (COPII)-coated vesicles and protein transport (Enninga et al., 2003) and acts as 1 of the 5 components in GTPase-activating protein target of rapamycin 2 complex, activating the 


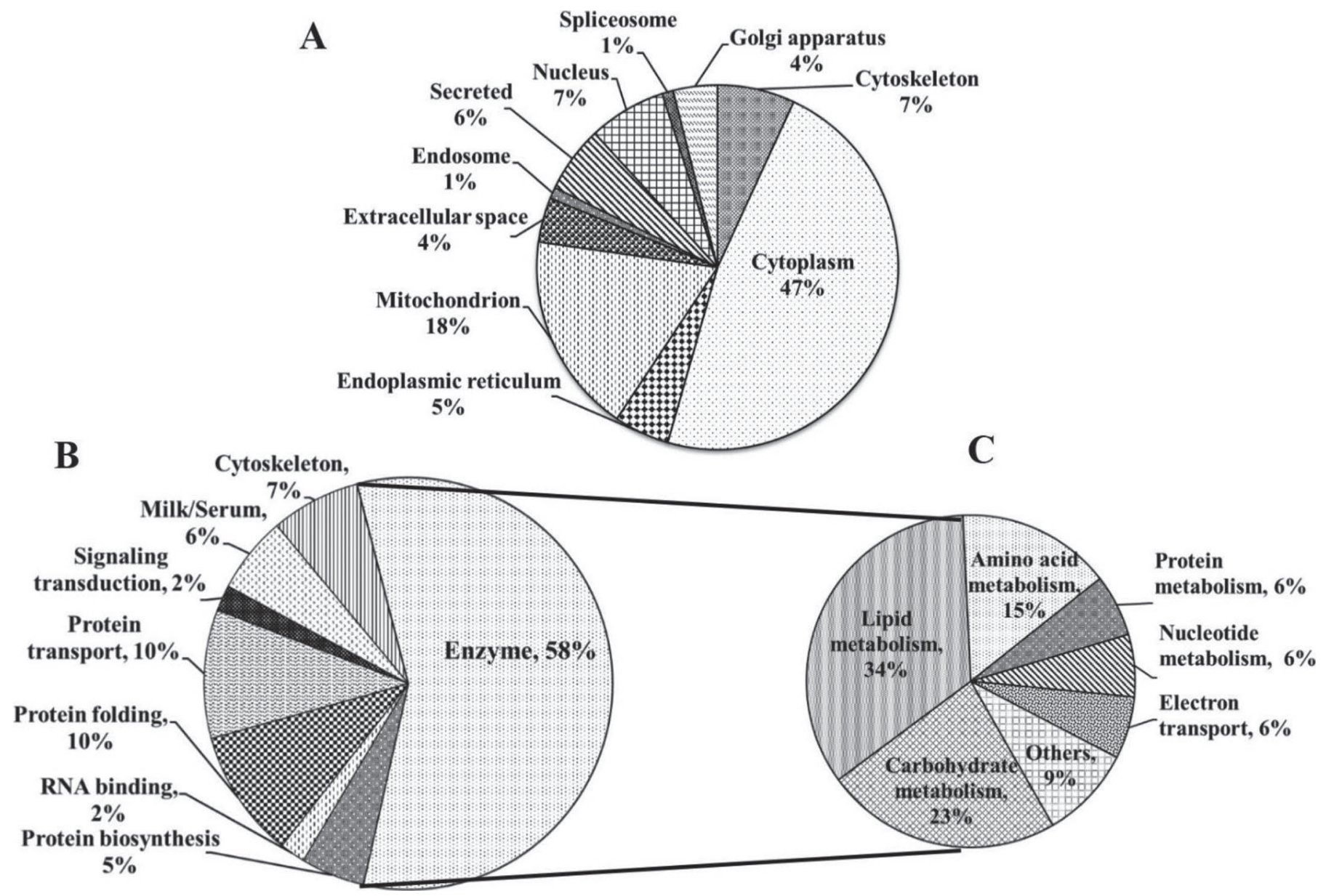

Figure 1. Classification of upregulated proteins in lactating and nonlactating mammary gland tissue based on subcellular location (A), biological function (B), and metabolism pathways of the enzymes (C).

mammalian target of rapamycin (mTOR) signaling pathway (Bar-Peled et al., 2013). Although preliminary findings based on a small number of cattle, these newly identified DEP might warrant further investigation to better understand their roles in regulating MG activity.

Functional analysis of the DEP in the lactating MG identified that most shifts were associated with macromolecular metabolism (Figure 1). In particular, enzymes, cytoskeleton, and protein processing (including protein biosynthesis, protein folding, and protein transport) were the most common processes associated with the DEP (Figure 1B). Some $57 \%$ of the upregulated DEP (32 proteins) had enzyme activity involved in metabolic pathways, including lipid (34\%), carbohydrate $(23 \%)$, AA (15\%), protein $(6 \%)$, and nucleotide $(6 \%)$ metabolism (Figure 1C). Differential expression of these functional pathways was also found in Bionaz and Loor (2011) and Rawson et al. (2012). Compared with the dry period, the 3 downregulated protein spots dur- ing lactation included WD repeat-containing protein 1 (WDR1; one cytoskeleton-associated protein), serotransferrin (TF; one iron-binding transport protein), and peroxiredoxin-6 (PRDX6). Notably, the enzyme PRDX6 prevents oxidative injury occurrence in the murine MG (Wang et al., 2003). Future work should attempt to better understand the role of PRDX6 in the nonlactating MG because it might be important in maintaining bovine mammary architecture before lactation.

Because milk production is highly energy dependent (McManaman and Neville, 2003), the activation of carbohydrate metabolism and the subsequent ATP synthesis are integrated to promote sufficient ATP availability for normal mammary function ( $\mathrm{Li}$ et al., 2016). Comparing the lactating and nonlactating tissues, the high abundance of 2 ATPase components [v-type proton ATPase subunit B and ATP synthase subunit $\beta$ (ATP5B)] and 2 mitochondrial enzymes 


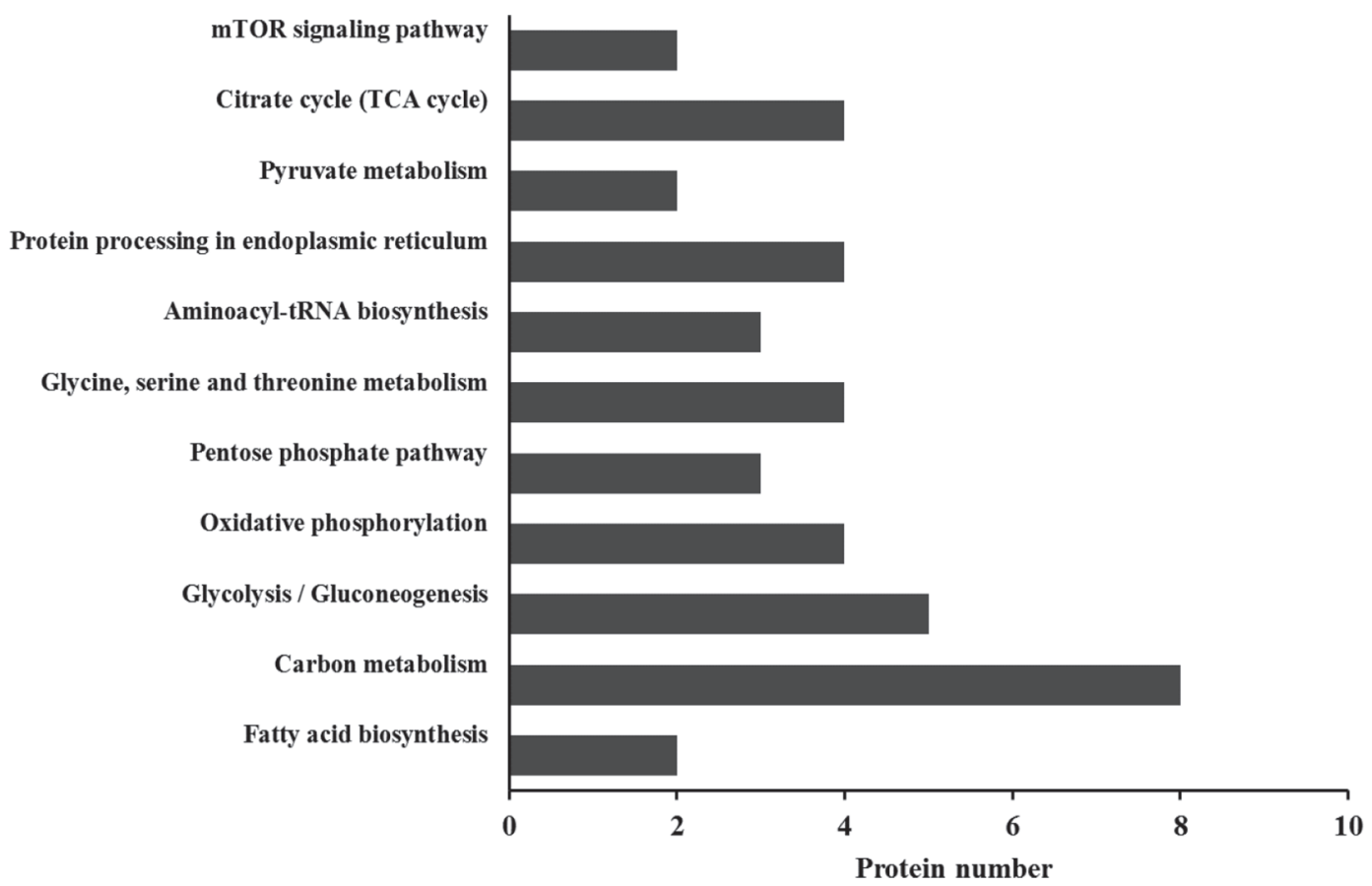

Figure 2. The representative Kyoto Encyclopedia of Genes and Genomes pathways of upregulated proteins in lactating and nonlactating mammary gland tissues. The $\mathrm{x}$-axis shows the number of proteins mapped to the certain pathway. $\mathrm{mTOR}=$ mammalian target of rapamycin; $\mathrm{TCA}=$ tricarboxylic acid.

[NADH-ubiquinone oxidoreductase subunit 1 and inorganic pyrophosphatase 1 (PPA1)] in the oxidative phosphorylation pathway (Figure 2 and Supplemental Table S2; https://doi.org/10.3168/jds.2016-12366) likely contributed to support this ATP demand by facilitating mitochondrial electron transport and oxidative phosphorylation process (Benit et al., 2001; Martin et al., 2005; Houreld et al., 2012). Similar changes of several ATPase subunits (including ATP5B and ATP5H) were also identified by Huang et al. (2014) and Zhao et al. (2015), who compared healthy and mastitic MG. Several upregulated DEP were significantly enriched in several carbohydrate metabolism-related pathways (such as carbon metabolism, glycolysis/gluconeogenesis, pyruvate metabolism, the tricarboxylic acid cycle, and the pentose phosphate pathway; Figure 2). For glycolysis/gluconeogenesis, 5 increased DEP [acetylCoA synthetase 1, fructose-bisphosphate aldolase C, dihydrolipoamide S-acetyltransferase-like, $\alpha$-enolase, and fructose-bisphosphatase 1] were critical for glucose metabolism during lactation. Specially, the enhanced level of acetyl-CoA synthetase 1 was consistent with its moderate transcript upregulation at the onset of lactation and increased gene/protein expression during lactation (Bionaz and Loor, 2011; Rawson et al., 2012). Another increased enzyme fructose-bisphosphatase 1 can catalyze the reaction of glycolysis from the third intermediate (fructose-1,6-bisphosphate) to pyruvate (Janjanam et al., 2014). In the tricarboxylic acid cycle, overexpression of isocitrate dehydrogenase 1 (cytoplasmic) would stimulate the conversion of isocitrate into oxalosuccinate to produce NADH for energy generation (Jena et al., 2015). Collectively, the increased proteins during lactation suggest mammary metabolism is altered toward more active carbohydrate metabolism and ATP synthesis to fulfill the energy and metabolite requirements for mammary cell proliferation and milk synthesis (Davies et al., 2006). A notable gap in upregulated DEP was in rate-limiting enzymes in lactose synthesis.

The above carbohydrate metabolism can provide some carbon-precursors linking lipid metabolism like glycerol-3-phosphate, which derives from glycolytic/ gluconeogenic intermediates stimulated by the upregulation of glycerol-3-phosphate dehydrogenase 1 (Beddek et al., 2008). The high production of glycerol-3-phosphate can be further used for synthesis of triglycerides from fatty acids through fatty acid synthase (Rudolph et al., 2007). The enhanced level of fatty acid synthase and glycerol-3-phosphate dehydrogenase 1 indicates glycerol-3-phosphate use in triglyceride synthesis is crucial during lactation, which agrees with overexpres- 
sion of the 2 genes involved in the enhanced level of mammary fatty acid synthesis during lactation (Bionaz and Loor, 2008).

The AA metabolism and milk protein synthesis are dynamic processes in lactating mammals (McManaman and Neville, 2003). Notably, the increased proteins 2-amino-3-ketobutyrate coenzyme A ligase and HIBADH are separately related to glycine synthesis (Marcus and Dekker, 1993) and L-valine degradation (Wanders et al., 2012) in lactating MG. To our knowledge, the function of mercaptopyruvate sulfur-transferase in MG is unknown. Milk protein synthesis should, in theory, be governed by AA availability. Noticeably, the 2 transfer RNA (tRNA) synthetases (seryl-tRNA synthetase and tryptophanyl-tRNA synthetase) were increased, signifying an enhanced level of seryl-/ tryptophanyl-tRNA synthesis, which likely supported the enhanced demands on protein synthesis machinery during lactation. With availability of AA substrates, protein translation proceeds in 3 phases: initiation, elongation, and termination. The enhanced abundance of 2 initiation factors (EIF2S1 and EIF3H) and one elongation factor (EEF1G) likely helps stimulate the initiation and elongation processes for peptide chain synthesis, consistent with their overexpression in lactating porcine mammary cells (Su et al., 2006) and bovine
MAC-T cells (Appuhamy et al., 2011). Also, eukaryotic translation elongation factor 2 and elongation factor 1-delta have been reported to increase during lactation in cattle (Bionaz and Loor, 2011; Jena et al., 2015). In contrast, the end-of-pathway genes for mTOR signaling initiation factors (eIF4B, eIF4E, and eukaryotic translation initiation factor $4 \mathrm{E}$ binding protein 1 ) that have frequently been reported to shift during lactation were not observed in this study. The different laboratory methods, different species of cows, and different stages in the lactating cycle may account for this.

As with other proteins, the process of protein folding plays a vital role in normal function performance for milk protein. As molecular chaperones, heat shock proteins (HSP) are vital in proper folding and translocation of polypeptides across membranes (De Maio, 1999). Importantly, the increased level of 3 HSP proteins, including HSPA4, HSPA9, and HSPD1 during lactation, indicate an extensive occurrence of protein folding. The upregulation of HSP27 has been observed during peak lactation in Bos taurus (Jena et al., 2015). In eukaryotic cells, protein folding before secretion takes place in the endoplasmic reticulum and protein exit from the endoplasmic reticulum requires COPII (Harding et al., 1999). The increased proteins SEC13 and SEC23 are 2 members of COPII complex consisting

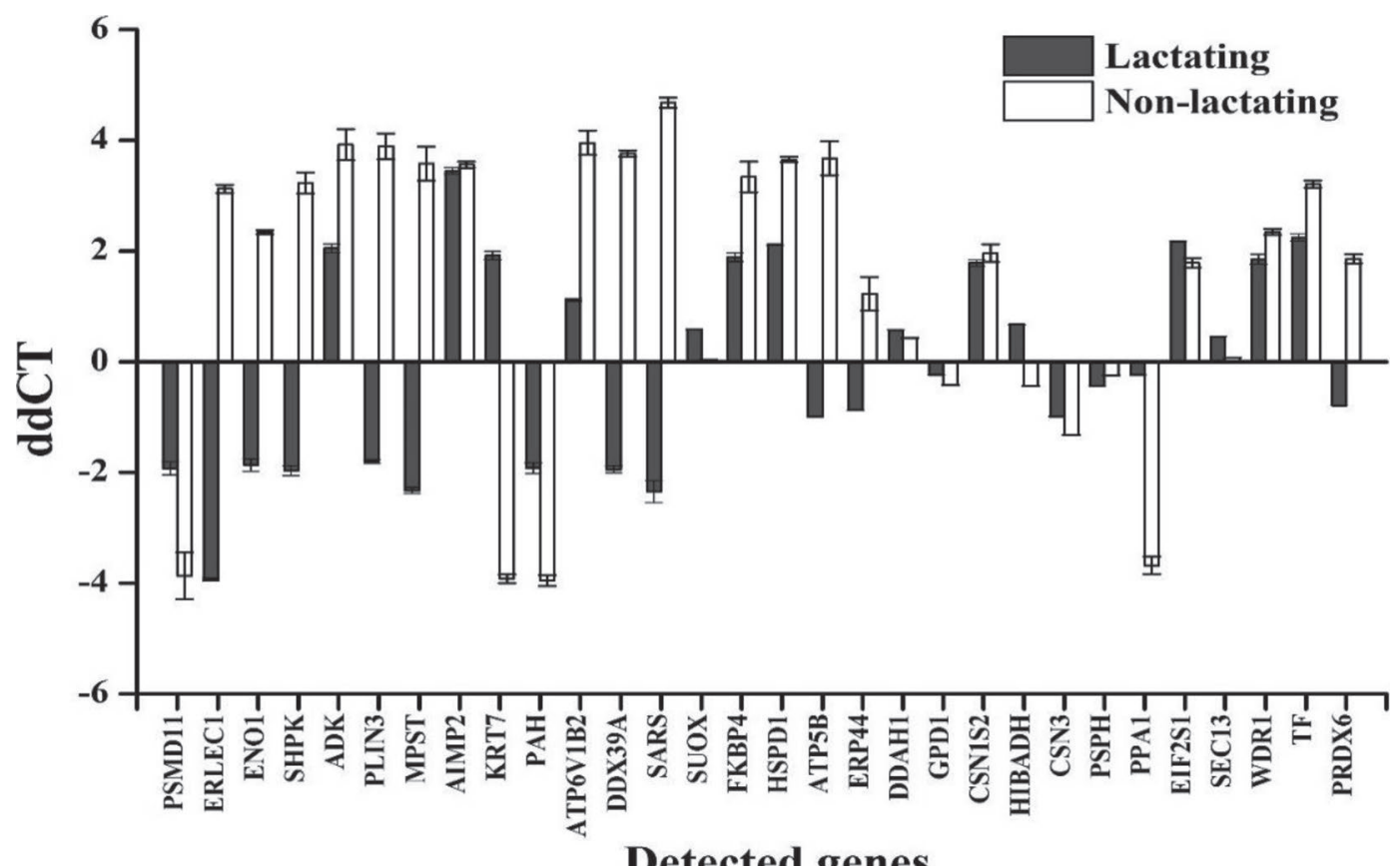

Figure 3. Transcriptional alteration of differentially expressed proteins in lactating and nonlactating mammary gland tissues. Total cellular RNA of lactating and nonlactating mammary gland tissue was analyzed by real-time, reverse-transcription PCR. Expressions in the samples were normalized to the $\beta$-actin. Error bars represent standard deviations. Gene names are listed in Supplemental Table S1 (https://doi. org/10.3168/jds.2016-12366). ddCT = (CTtarget gene-CTß-actin) lactating group, (CTtarget gene-CT $\beta$-actin) nonlactating group. 
of 5 components (GTP-binding protein SAR1, SEC23, SEC24, SEC13, and SEC31). A high level of SEC23B expression has been observed in secretory tissues of lactating cows (Beddek et al., 2008), which may further facilitate milk secretion.

Thirty proteins were selected for RT-PCR analysis (Figure 3), among which the mRNA abundance of 22 genes were significantly expressed. As expected, the 3 downregulated proteins (WDR1, PRDX6, and TF) showed a significant decrease consistent with their mRNA abundance. Similarly, 16 of the upregulated genes were in the same expression pattern of DEP; however, mRNA expression of phenylalanine-4-hydroxylase, keratin 7 , and PPA1 was decreased inconsistent with the upregulation identified in the 2-D proteome analysis. Because only 3 dairy cows in each group were used in this study, the results have low power and should be interpreted with caution. This low power may have contributed to the poor agreement between the 2-D data and the RT-PCR results. Moreover, 2 milk synthesis-related proteins, CSN3 and EIF2S1, were selected for Western blot analysis (Figure 4) and their expression were both significantly increased in lactating cows compared with those in the nonlactating cows $(P$ $<0.05)$. The results further confirmed the concordance of proteins CSN3 and EIF2S1 with those observed in RT-PCR and difference gel electrophoresis.

Overall, our study shows the differential abundance of 57 mammary cellular and 3 secreted proteins involved in various metabolic metabolisms in the lactating MG comparing dry MG. Five proteins (IMMT, NANS, HIBADH, ERLEC1, SEC13, and DDAH1) were not

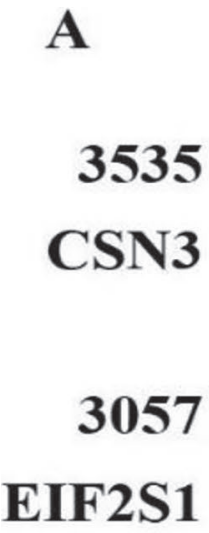

C
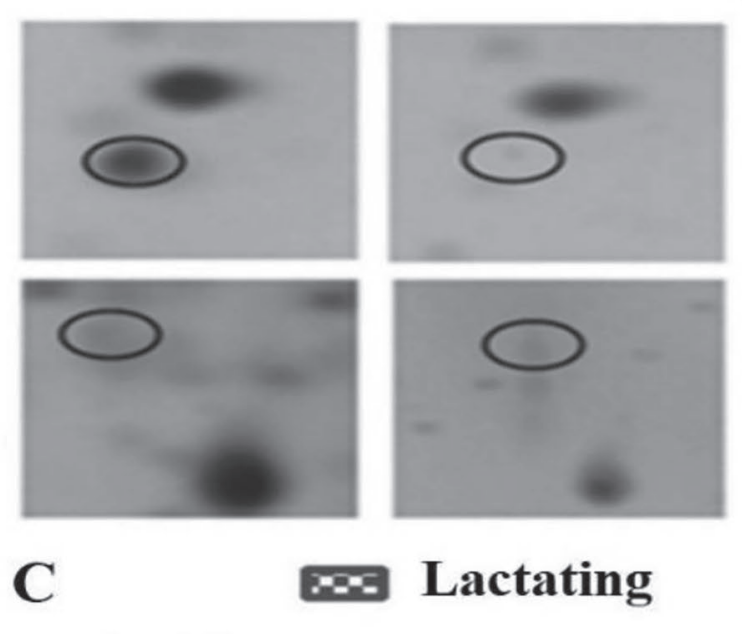

Lactating
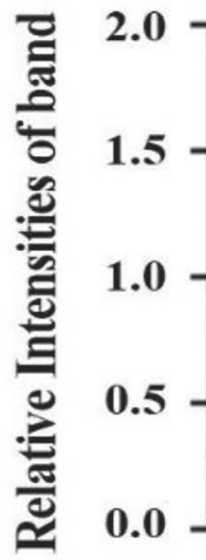

B
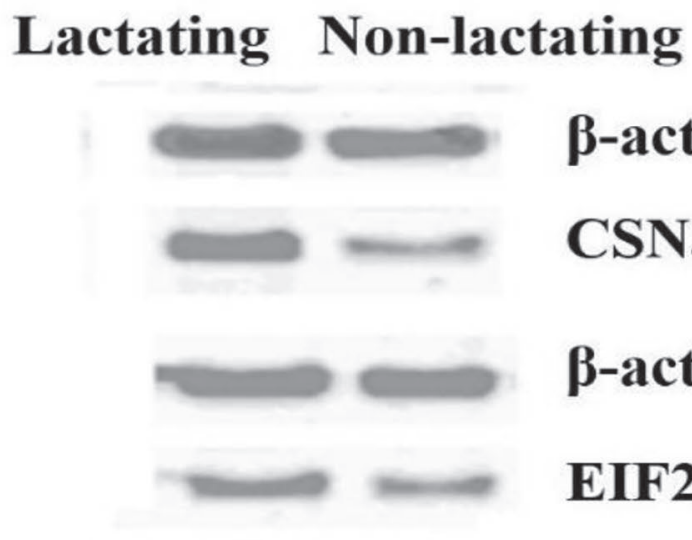

$\beta$-actin

CSN3

B-actin

EIF2S1
288 Non-lactating

$* *$

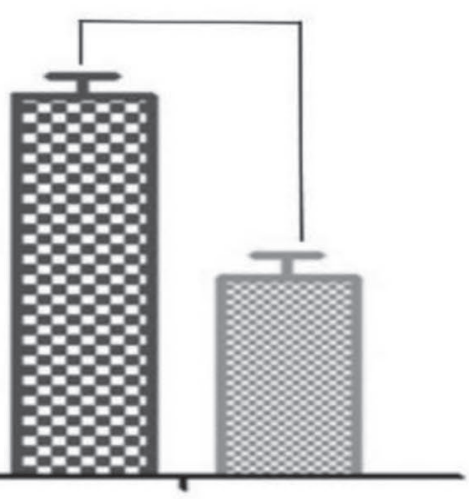

EIF2S1

Figure 4. Kappa-casein (CSN3) and eukaryotic translation initiation factor 2 subunit 1 (EIF2S1) expression in lactating and nonlactating mammary gland tissues. (A) The expression changes of CSN3 and EIF2S1 in 2-dimensional electrophoresis gels. (B) Western blot of CSN3 and EIF2S1 protein expression. (C) The densitometric intensity of immunoblots. $\beta$-actin served as the internal control. Error bars represent SD. $* P$ $<0.05,{ }^{* *} P<0.01$. 
previously identified related to MG lactating processes. All these DEP were primarily involved in a wide range of molecular metabolic processes that are crucial for milk production and MG maintenance. Although conducted on a limited number of animals, this study provides targets for future work elucidating factors critical to MG involution, maintenance, and function.

\section{ACKNOWLEDGMENTS}

This research was supported by grants from the National Key Research and Development Program of China (2016YFD0500503), National Natural Science Foundations of China (31672447, 31372336).

\section{REFERENCES}

Appuhamy, J. A., A. L. Bell, W. A. Nayananjalie, J. Escobar, and M. D. Hanigan. 2011. Essential amino acids regulate both initiation and elongation of mRNA translation independent of insulin in MAC-T cells and bovine mammary tissue slices. J. Nutr. 141:1209-1215.

Bar-Peled, L., L. Chantranupong, A. D. Cherniack, W. W. Chen, K. A. Ottina, B. C. Grabiner, E. D. Spear, S. L. Carter, M. Meyerson, and D. M. Sabatini. 2013. A Tumor suppressor complex with GAP activity for the Rag GTPases that signal amino acid sufficiency to mTORC1. Science 340:1100-1106.

Beddek, A. J., P. Rawson, L. Peng, R. Snell, K. Lehnert, H. E. Ward, and T. W. Jordan. 2008. Profiling the metabolic proteome of bovine mammary tissue. Proteomics 8:1502-1515.

Bellmann, O. 1976. Hormonal regulation of lactation. Klin. Padiatr. 188:385-395.

Benit, P., D. Chretien, N. Kadhom, P. de Lonlay-Debeney, V. Cormier-Daire, A. Cabral, S. Peudenier, P. Rustin, A. Munnich, and A. Rotig. 2001. Large-scale deletion and point mutations of the nuclear NDUFV1 and NDUFS1 genes in mitochondrial complex I deficiency. Am. J. Hum. Genet. 68:1344-1352.

Bionaz, M., and J. J. Loor. 2008. Gene networks driving bovine milk fat synthesis during the lactation cycle. BMC Genomics 9:366.

Bionaz, M., and J. J. Loor. 2011. Gene networks driving bovine mammary protein synthesis during the lactation cycle. Bioinform. Biol. Insights 5:83-98.

Bradford, M. M. 1976. A rapid and sensitive method for the quantitation of microgram quantities of protein utilizing the principle of protein-dye binding. Anal. Biochem. 72:248-254.

Breckenridge, R. A., P. Kelly, M. Nandi, P. J. Vallance, T. J. Ohun, and J. Leiper. 2010. A role for Dimethylarginine Dimethylaminohydrolase 1 (DDAH1) in mammalian development. Int. J. Dev. Biol. 54:215-220.

Collier, R. J., E. L. Annen-Dawson, and A. Pezeshki. 2012. Effects of continuous lactation and short dry periods on mammary function and animal health. Animal 6:403-414

Davies, C. R., J. S. Morris, M. R. Griffiths, M. J. Page, A. Pitt, T. Stein, and B. A. Gusterson. 2006. Proteomic analysis of the mouse mammary gland is a powerful tool to identify novel proteins that are differentially expressed during mammary development. Proteomics 6:5694-5704.

De Maio, A. 1999. Heat shock proteins: Facts, thoughts, and dreams. Shock 11:1-12.

Desrivieres, S., K. Kuhn, J. Muller, M. Glaser, N. C. P. Laria, J. Kordet, M. Sonnentag, T. Neumann, J. Schwarz, J. Schafer, C. Hamon, B. Groner, and T. Prinz. 2007. Comparison of the nuclear proteomes of mammary epithelial cells at different stages of functional differentiation. Proteomics 7:2019-2037.
Dufour, J. J., and G. L. Roy. 1985. Distribution of ovarian follicular populations in the dairy cow within 35 days after parturition. J. Reprod. Fertil. 73:229-235.

Enninga, J., A. Levay, and B. M. Fontoura. 2003. Sec13 shuttles between the nucleus and the cytoplasm and stably interacts with Nup96 at the nuclear pore complex. Mol. Cell. Biol. 23:7271-7284.

Harding, H. P., Y. Zhang, and D. Ron. 1999. Protein translation and folding are coupled by an endoplasmic-reticulum-resident kinase. Nature 397:271-274

Houreld, N. N., R. Masha, and H. Abrahamse. 2012. Profiling of genes central to human mitochondrial energy metabolism following low intensity laser irradiation. AIP Conf. Proc. 1486:53-62.

Huang, J., G. Luo, Z. Zhang, X. Wang, Z. Ju, C. Qi, Y. Zhang, C. Wang, R. Li, J. Li, W. Yin, Y. Xu, S. J. Moisa, J. J. Loor, and J. Zhong. 2014. iTRAQ-proteomics and bioinformatics analyses of mammary tissue from cows with clinical mastitis due to natural infection with Staphylococci aureus. BMC Genomics 15:839.

Hurley, W. L. 1989. Mammary gland function during involution. J Dairy Sci. 72:1637-1646.

Janjanam, J., S. Singh, M. K. Jena, N. Varshney, S. Kola, S. Kumar, J. K. Kaushik, S. Grover, A. K. Dang, M. Mukesh, B. S. Prakash, and A. K. Mohanty. 2014. Comparative 2D-DIGE proteomic analysis of bovine mammary epithelial cells during lactation reveals protein signatures for lactation persistency and milk yield. PLoS One 9:e102515.

Jena, M. K., J. Janjanam, J. Naru, S. Kumar, S. Kumar, S. Singh, S. K. Mohapatra, S. Kola, V. Anand, S. Jaswal, A. K. Verma D. Malakar, A. K. Dang, J. K. Kaushik, V. S. Reddy, and A. K. Mohanty. 2015. DIGE based proteome analysis of mammary gland tissue in water buffalo (Bubalus bubalis): Lactating vis-a-vis heifer. J. Proteomics 119:100-111.

Li, C., W. Cai, C. Zhou, H. Yin, Z. Zhang, J. J. Loor, D. Sun, Q. Zhang, J. Liu, and S. Zhang. 2016. RNA-Seq reveals 10 novel promising candidate genes affecting milk protein concentration in the Chinese Holstein population. Sci. Rep. 6:26813.

Li, Z., H. Liu, X. Jin, L. Lo, and J. Liu. 2012. Expression profiles of microRNAs from lactating and non-lactating bovine mammary glands and identification of miRNA related to lactation. BMC Genomics 13:731.

Marcus, J. P., and E. E. Dekker. 1993. Threonine formation via the coupled activity of 2-amino-3-ketobutyrate coenzyme-A lyase and threonine dehydrogenase. J. Bacteriol. 175:6505-6511.

Martin, M. A., A. Blazquez, L. G. Gutierrez-Solana, D. FernandezMoreira, P. Briones, A. L. Andreu, R. Garesse, Y. Campos, and J. Arenas. 2005. Leigh syndrome associated with mitochondrial complex I deficiency due to a novel mutation in the NDUFS1 gene. Arch. Neurol. Chicago 62:659-661.

McManaman, J. L., and M. C. Neville. 2003. Mammary physiology and milk secretion. Adv. Drug Deliv. Rev. 55:629-641.

Mishima, T., T. Hamada, K. Ui-Tei, F. Takahashi, Y. Miyata, J. Imaki, H. Suzuki, and K. Yamashita. 2004. Expression of DDAH1 in chick and rat embryos. Brain Res. Dev. Brain Res. 148:223-232.

Rawson, P., C. Stockum, L. Peng, B. Manivannan, K. Lehnert, H. E. Ward, S. D. Berry, S. R. Davis, R. G. Snell, D. McLauchlan, and T. W. Jordan. 2012. Metabolic proteomics of the liver and mammary gland during lactation. J. Proteomics 75:4429-4435.

Rudolph, M. C., J. L. McManaman, T. Phang, T. Russell, D. J. Kominsky, N. J. Serkova, T. Stein, S. M. Anderson, and M. C. Neville. 2007. Metabolic regulation in the lactating mammary gland: A lipid synthesizing machine. Physiol. Genomics 28:323-336.

Schmittgen, T. D., and K. J. Livak. 2008. Analyzing real-time PCR data by the comparative C(T) method. Nat. Protoc. 3:1101-1108.

Su, Z., X. Dong, B. Zhang, Y. Zeng, Y. Fu, J. Yu, and S. Hu. 2006. Gene expression profiling in porcine mammary gland during lactation and identification of breed- and developmental-stage-specific genes. Sci. China C Life Sci. 49:26-36.

Suarez-Vega, A., B. Gutierrez-Gil, C. Klopp, G. Tosser-Klopp, and J. J. Arranz. 2016. Comprehensive RNA-Seq profiling to evaluate lactating sheep mammary gland transcriptome. Sci. Data 3:160051. 
Wanders, R. J. A., M. Duran, and F. J. Loupatty. 2012. Enzymology of the branched-chain amino acid oxidation disorders: The valine pathway. J. Inherit. Metab. Dis. 35:5-12.

Wang, D., G. Liang, B. Wang, H. Sun, J. Liu, and L. L. Guan. 2016. Systematic microRNAome profiling reveals the roles of microRNAs in milk protein metabolism and quality: Insights on low-quality forage utilization. Sci. Rep. 6:21194.

Wang, X., S. A. Phelan, K. Forsman-Semb, E. F. Taylor, C. Petros, A. Brown, C. P. Lerner, and B. Paigen. 2003. Mice with targeted mutation of peroxiredoxin 6 develop normally but are susceptible to oxidative stress. J. Biol. Chem. 278:25179-25190.

Wu, Y., C. Peng, L. Xu, X. Zheng, M. Liao, Y. Yan, Y. Jin, and J. Zhou. 2012. Proteome dynamics in primary target organ of infectious bursal disease virus. Proteomics 12:1844-1859.

Yang, J., J. J. Kennelly, and V. E. Baracos. 2000. The activity of transcription factor Stat5 responds to prolactin, growth hormone, and IGF-I in rat and bovine mammary explant culture. J. Anim. Sci. 78:3114-3125.

Yang, J. X., C. H. Wang, Q. B. Xu, F. Q. Zhao, J. X. Liu, and H. Y. Liu. 2015. Methionyl-methionine promotes alpha-s1 casein synthesis in bovine mammary gland explants by enhancing intracellular substrate availability and activating JAK2-STAT5 and mTORmediated signaling pathways. J. Nutr. 145:1748-1753.

Zhao, K., H. Y. Liu, M. M. Zhou, and J. X. Liu. 2010. Establishment and characterization of a lactating bovine mammary epithelial cell model for the study of milk synthesis. Cell Biol. Int. 34:717-721.

Zhao, X. W., Y. X. Yang, D. W. Huang, G. L. Cheng, and H. L. Zhao. 2015. Comparative proteomic analysis of proteins expression changes in the mammary tissue of cows infected with Escherichia coli mastitis. J. Vet. Sci. 16:253-263. 